\title{
LA AGENDA 2030 Y LOS DESAFÍOS PARA LA EDUCACIÓN SUPERIOR
}

\author{
The 2030 Agenda and the challenges for higher education
}

\author{
Emilce Sena Correa ${ }^{1}$
}

\begin{abstract}
Resumen: La educación para el desarrollo sostenible precisará de un nuevo paradigma educativo, principalmente, que responda a los diferentes cambios: económicos, sociales, políticos y culturales. Educar para el desarrollo sostenible de los países, deberá entoncesestar enfocado hacía el desarrollo del conocimiento. Las instituciones de educación superior tienen como función sustantiva la docencia, la investigación y la extensión. A su vez las IES tienen el compromiso de encontrar la solución de la problemática regional, entre otros, problemas ambientales, derivados del cambio climático. Este trabajo presenta el papel que la universidad, que debe tener como actor fundamental de la sociedad, la búsqueda de alcanzar los Objetivos de Desarrollo Sostenible. En cuanto al método utilizado, es investigación documental realizando una revisión sistemática cualitativa de documentos publicados en la última década con el propósito de establecer relaciones, diferencias, etapas, posiciones o estado actual del conocimiento respecto al tema objeto de estudio en algunos países de América Latina.Se cierra concluyendo que la Educación Superior, es clave en el desarrollo sustentable para América Latina.
\end{abstract}

Palabras claves: Agenda 2030 PNUD, Educación Superior América Latina, ODS.

\begin{abstract}
Education for sustainable development will require a new educational paradigm, mainly, that responds to the different changes: economic, social, political and cultural. Educate for the sustainable development of the countries, must be focused towards the development of knowledge. Higher education institutions have as substantive functions teaching, research and extension. In turn, Hight Education Institutions are committed to finding the solution to regional problems, among others, environmental problems, derived from climate change. This paper presents the role that the university must have as a fundamental actor in society, the search to achieve the Sustainable Development Goals. Regarding the method used, this research is documentary, conducting a qualitative systematic review of documents published in the last decade, with the purpose of establishing relationships, differences, stages, positions or current state of knowledge regarding the subject under study in some Latin American countries. It closes concluding that Higher Education is the key in for Latin America development.
\end{abstract}

Keywords: 2030 United Nations Agenda, Latin America Education, Sustentable Development Goals.

\footnotetext{
${ }^{1}$ Facultad Politécnica, Universidad Nacional de Asunción, Paraguay
} 


\section{Introducción}

El ODS Número 4, parte de los 17 ODS, referente a la Educación como un todo, y la meta 4.3 sí corresponde a la Educación Superior -interés de este trabajo, reza lo siguiente, "Para el 2030, asegurar el acceso en condiciones de igualdad para todos los hombres y las mujeres a una formación técnica, profesional y superior de calidad, incluida la enseñanza universitaria". (UNESCO). Partamos entonces, afirmando que la educación superior, es un elemento fundamental para alcanzar los ODS.

Así, el Programa Pacto Global, iniciativa promovida por las Naciones Unidas, en la cual las empresas y organizaciones se comprometen a alinear sus estrategias y operaciones con diez principios universalmente aceptados en cuatro áreas temáticas como: derechos humanos y laborales, medio ambiente y la corrupción. (Pacto Global, Paraguay). Éste busca cumplir con la Agenda 2030, basada en los 17 ODS, aprobados en la Asamblea General de la ONU, en el cual más de 150 Jefes de Estado y de Gobierno reunidos en el 2015, y aprobaron la Agenda 2030. Desde enero de 2016, rigen los esfuerzos de los países, para lograr un mundo sostenible en el año 2030. El informe de la Cumbre de La Antigua, Guatemala, reafirma la importancia de la Agenda 2030, para afianzar un cambio de paradigma hacia un desarrollo más sostenible, en la comunidad iberoamericana.

Así también la Organización Internacional de Universidades por el Desarrollo Sostenible y el Medio Ambiente (OIUDSMA), integrada por 59 universidades, en el Congreso (2004), estableció que su principal compromiso, consiste en el replanteamiento de las funciones esenciales de la Educación Superior: la docencia, la investigación, la extensión y la gestión, con el objetivo de configurar una nueva cultura institucional, que se oriente por los principios del Desarrollo Sostenible.

Se plantea que las universidades, según lo que mecionan, Mader, 2012; Tilbury, 2012 y Fadeeva et.al., citado por Alba Hidalgo, 2015, para ser sostenibles, las universidades han de ser transformadoras. Existe un cierto consenso internacional sobre el rol que deben cumplir las universidades en relación a la sostenibilidad, tal y como reflejan las más de treinta declaraciones, cartas o iniciativas internacionales que han sido aprobadas, difundidas y firmadas por más de 1.400 representantes de las universidades en los últimos treinta años. (Wright, 2004; Grindsted, 2011; Grindsted y Holm, 2012, y Lozano et. al., citado por Alba Hidalgo).

En este sentido, la educación superior se convierte en un elemento clave para asegurar que los procesos formativos de los ciudadanos del futuro, incorporen entre sus líneas de actuación esta mirada holística y crítica pues, como señalan Sáenz-Rico, Benítez, Neira, Sobrino y D’angelo, citado en Revisión sistemática sobre competencias en desarrollo sostenible en educación superior, de Valentín Gonzalo Muñoz et. al., "es uno de los mayores retos que se debe plantear la 
universidad para participar, desde la formación docente, en la construcción de sociedades sostenibles".

La Meta de la Agenda 2030, por lo tanto, la Educación debe ser su estandarte de presentación...

La Agenda 2030, es no dejar a ninguna persona sin educación, sin embargo, eso no está ocurriendo. El Informe Mundial de la UNESCO, demuestra que más 264.000.000 millones están fuera de las escuelas y que la mayoría son niñas. Muchas son las barreras, con las que se encuentran las mujeres para acceder a la educación, en su rol de investigadoras, de trabajadoras... si no se eliminan esas barreras, será difícil lograr el Desarrollo Sostenible, principalmente, el Objetivo 4. (Instituto de Estadística, Unesco)

El objetivo número 4 de los ODS, es claro al respecto, cuando señala: "Garantizar una educación inclusiva, equitativa y de calidad y promover oportunidades de aprendizaje durante toda la vida para todos". Asimismo el 4.7 de los ODS, centra su eje de atención en la importancia de la educación para la sostenibilidad. Lo expresa en los siguientes términos, para el 2030, garantizar que todos los alumnos adquieran los conocimientos teóricos y prácticos necesarios para promover el desarrollo sostenible, entre otras cosas mediante la educación para el desarrollo sostenible y la adopción de estilos de vida sostenibles, los derechos humanos, la igualdad entre los géneros, la promoción de una cultura de paz y no violencia, la ciudadanía mundial y la valoración de la diversidad cultural y de la contribución de la cultura al desarrollo sostenible, entre otros medios. (Unesco)

Para alcanzar los ODS, todos los países -principalmente, los de América Latina-, deben emprender transformaciones importantes en la educación, la salud, los sistemas de energía, el uso de la tierra, el desarrollo urbano y muchas otras. Cada transformación requiere políticas públicas con acciones a largo plazo, que involucren a los actores del gobierno, las empresas y de la sociedad civil. Estas transformaciones no podrán ser impulsadas por el propio mercado, sino desde los mismos gobiernos deben surgir las iniciativas para movilizar a las partes interesadas, planificar y ejecutar las transformaciones, diseñar procesos de políticas de apoyo y aplicar el financiamiento público.

Para los países de América Latina y el Caribe, la situación se torna compleja por los efectos que ha generado las políticas neoliberales durante los últimos 30 años en los países de la región, así:

- De los 17 ODS, 5 de ellos por lo menos ha logrado alcanzar el máximo valor de la variable analizada (verde).

- El 5 \% de los ODS de todos los países han logrado alcanzar el máximo valor de la variable analizada (verde).

- $\quad$ El $63 \%$ de los ODS de todos los países se encuentra en una brecha muy distante para alcanzar los ODS (rojo \&naranja). (Ojeda Suárez, R., 2019) 
Gráfico 1: Índice y Ranking Avances ODS 2030 países de América Latina y el Caribe

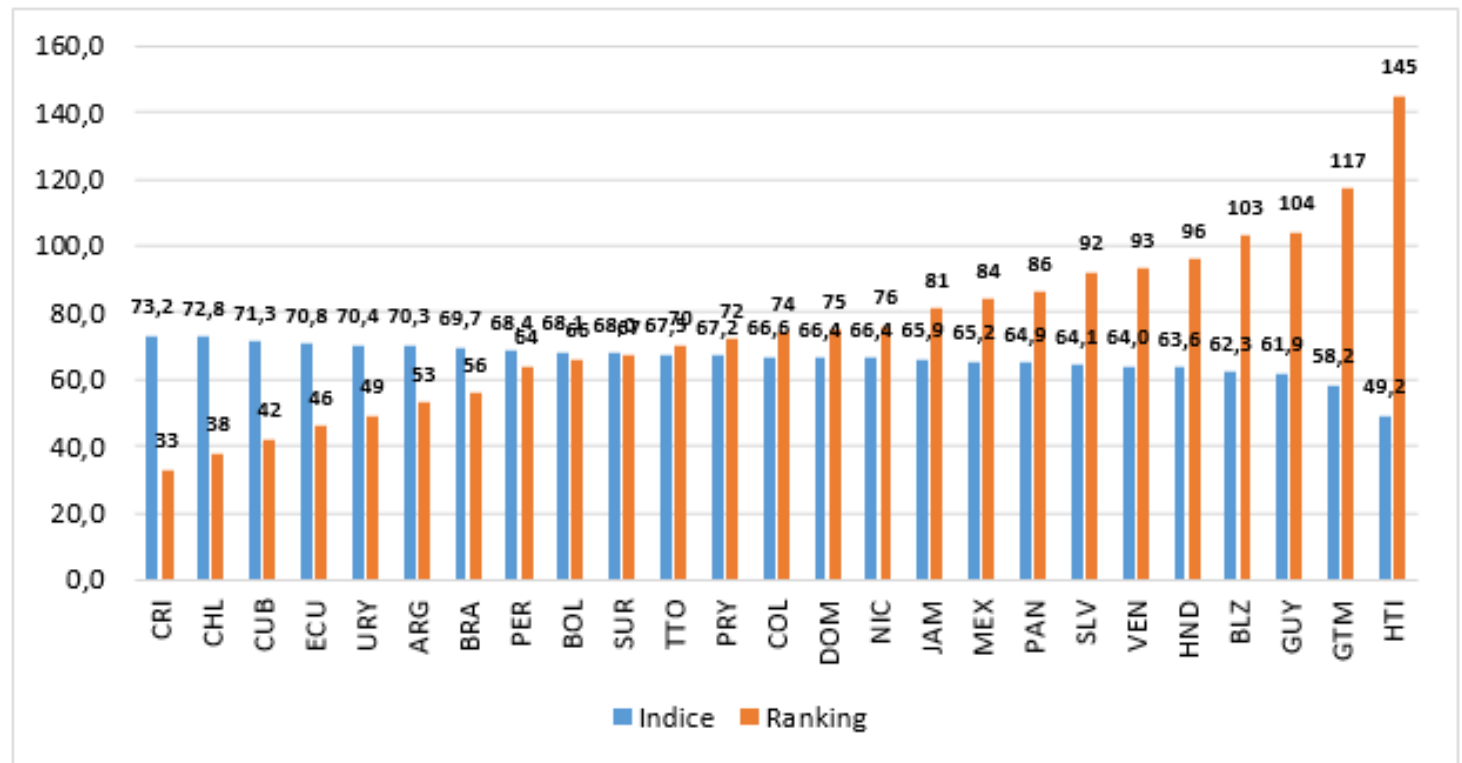

Fuente: Ojeda Suárez, R., 2019

En los últimos 10 años se han desarrollado metodologías para evaluar los avances de los países relacionados con el medio ambiente a través del Índice de Sostenibilidad Ambiental EPI (Environmental Performance Index). El EPI fue precedido por el Índice de Sustentabilidad Ambiental (ESI), publicado entre 1999 y 2005. Ambos índices fueron desarrollados por la Universidad de Yale (Yale Center for Environmental Law and Policy) y la Universidad de Columbia (Center for International Earth Science Information Network) en colaboración con el Foro Económico Mundial y el Centro de Investigación de la Comisión Europea. Para evaluar el progreso de un país en un indicador en particular, se considera cuatro bandas: la banda verde está limitada por el máximo que se pueden lograr para cada variable. Las tres bandas de color que van del amarillo al naranja y al rojo denotan una distancia creciente del logro de los ODS. (Ojeda Suárez, R., 2019)

Por otra parte, la Secretaria Ejecutiva de la CEPAL, Alicia Bárcena (2019), presentó un informe durante el Foro de los Países de América Latina 2019, que incluyó proyecciones al 2030 de 20 indicadores de avance en 15 de los ODS en América Latina y el Caribe. En primer lugar, según Bárcena, ya se alcanzó la meta en la reducción de la tasa de mortalidad de niños menores de 5 años al menos a 25 por cada 1.000 nacidos vivos (meta 3.2). Segundo, se aprecia una tendencia correcta en el porcentaje de personas que usan Internet (meta 17.8). Pero sin embargo, señala la misma que no se alcanzará la meta de lograr el acceso universal y equitativo al agua potable a un precio asequible para todos (meta 6.1). Por otro lado, la tendencia en el acceso a la educación secundaria no es suficiente para que todos culminen el nivel oportunamente en el 2030 (meta 4.1) y se frenó la tendencia positiva en la baja en el porcentaje de la prevalencia de la 
subalimentación (meta 2.1). En tanto, el desempeño económico y distributivo es insuficiente para eliminar la pobreza extrema: en los últimos 10 años, el PIB de América Latina ha crecido a menos del $2 \%$ anual $(1,7 \%)$.

Pascual Gerstenfeld, director de la División de Estadísticas de la CEPAL, señala que "La Agenda 2030 y los ODS no son meramente un programa estadístico global; es un programa que tiene una agenda política de cooperación" para cambiar el modelo de desarrollo actual, indica.

\section{Desarrollo Sostenible en Paraguay}

"Paraguay tiene el compromiso de una agenda para erradicar la pobreza, el desarrollo en la salud y en el medio ambiente. Es decir, un desarrollo sostenible sin comprometer los recursos para las generaciones futuras, ya que existe un solo planeta y ese planeta hay que cuidarlo", indicó el coordinador de asuntos ambientales de la Cancillería Nacional y actual coordinador de la Comisión de Objetivos de Desarrollo Sostenible (ODS) Paraguay 2030. La Comisión ODS Paraguay (creada por decreto del Poder Ejecutivo №5887 del 6 de setiembre del 2016), está coordinada por el Ministerio de Relaciones Exteriores.

En Paraguay, una importante meta del proyecto es que la iniciativa sea una contribución concreta y efectiva al cumplimiento de los Objetivos de Desarrollo del Milenio por parte del empresariado paraguayo; y que a su vez ésta, constituya la adhesión formal y explícita a los principios consagrados en el Pacto Global.

El PNUD Paraguay, en alianza con otras organizaciones empresariales, inició un proceso para facilitar y estrechar vínculos entre los sectores social, público y privado. El grupo impulsor, liderado por 26 organizaciones paraguayas, se han comprometido a promover, difundir, establecer y fortalecer prácticas en responsabilidad social que conduzcan a reducir brechas en relación directa a los indicadores vinculados a los ODM. Es así, como en el año 2008, se lanzó oficialmente la Red local del Pacto Global en Paraguay. (Pacto Global, Paraguay).

Actualmente, están adheridos a la iniciativa; Empresas, ONG’s, Cámaras, Asociaciones, Academia, Gobernaciones y Municipios.

El papel que la Academia, como actor fundamental de la sociedad, velará por el cumplimiento y las estrategias de búsqueda para alcanzar los Objetivos de Desarrollo Sostenible

Así en el caso de la Universidad Nacional de Asunción, en setiembre de 2019, se realizó el Conversatorio Juventudes 2030 Paraguay y el lanzamiento del Programa de Fortalecimiento ODS, Juventudes Paraguay 2030. El objetivo del mismo fue generar capacidades y sinergias que potencien el protagonismo de los 
jóvenes, de entre 18 y 35 años, en torno a los ODS, teniendo en cuenta que constituyen la población mayoritaria en Paraguay y por consiguiente actores claves en la implementación de la Agenda 2030 en el Paraguay. En el mismo marco, se llevó a cabo la firma de un memorando de entendimiento entre la UNA y la Comisión ODS.

Durante el evento la Rectora de la Universidad Nacional de Asunción, hizo mención al memorando de entendimiento suscripto entre la UNA y la Comisión ODS, y señaló que "como universidad, estamos empeñados en la formación científica y humana de los jóvenes, en el marco de una innovación permanente y la actualización tecnológica para adecuar la capacidad de los estudiantes a un mercado cada vez más globalizado". Añadió, "Estamos seguros de que la academia, mediante la aplicación de los conocimientos será siempre el faro que nos guíe hacia un crecimiento sostenido y sustentable para el bienestar de todos los ciudadanos", indicó.

En Paraguay, a través del Programa Pacto Global, Capítulo Paraguay, se espera que en los próximos años, las reformas curriculares de las carreras de grado y programas de postgrado, inserten contenidos sobre desarrollo sustentable, así también, la inclusión de la sostenibilidad como criterio de calidad en la evaluación institucional de las universidades, por medio de las agencias acreditadoras.

\section{Conclusiones}

La Universidad en virtud de sus ejes misionales: la docencia, la investigación y la extensión, deben contribuir a reducir la pobreza extrema, mejorar las condiciones del medio ambiente y promover un acceso de la población a condiciones de vida más dignas.

La contribución de la investigación universitaria a los ODS, será formar a los estudiantes en la investigación del desarrollo sostenible. La responsabilidad social universitaria, NO puede estar ajena al cumplimiento de las metas de desarrollo sostenible.

A nivel regional, se hace necesaria de una alianza global, a partir de la consolidación de los espacios regionales ya existentes (ANUIES), con el propósito de analizar y discutir, desde una perspectiva global la educación superior, la ciencia, tecnología e innovación, las políticas y estrategias que deben adoptar los países para el logro de los ODS.

Por último, la educación, debe ser una prioridad para todos los gobiernos, asignando el 7\% del PIB, y $15 \%$ a $20 \%$ del Gasto Público total a la misma. 


\section{Referencias}

Agencia EFE, Santiago Chile. Países de América Latina evalúan Objetivos de Desarrollo Sostenible (ODS) de 2030. Disponible en https://www.efe.com/efe/cono-sur/destacada-cono-sur/paises-de-americalatina-evaluan-objetivos-desarrollo-sostenible-ods-2030/50000832-3770129

ALBA HIDALGO, David. (2017). Hacia una fundamentación de la sostenibilidad en la educación superior. Revista Iberoamerica de Educación. (73) Disponible en https://rieoei.org/RIE/article/view/197

BÁRCENA, Alicia. (2019). Cumplimiento de la Agenda 2030 en América Latina y el Caribe se encuentra en un punto crítico. Disponible en https://www.cepal.org/es/noticias/cumplimiento-la-agenda-2030-americalatina-caribe-se-encuentra-un-punto-critico-alicia

CASTIZO ROBLES, Rosa. (2019). Iberoamérica, en un momento decisivo para lograr un desarrollo sostenible. Disponible en https://www.somosiberoamerica.org/tribunas/iberoamerica-en-un-momentodecisivo-para-lograr-un-desarrollo-sostenible/

CEPAL. (2019). Informe de avance cuatrienal sobre el progreso y los desafíos regionales de la Agenda 2030 para el Desarrollo Sostenible en América Latina y el Caribe. Disponible en https://repositorio.cepal.org/handle/11362/44551

GONZALO MUÑON, Valentín. et. al. Revisión sistemática sobre competencias en desarrollo sostenible en educación superior. Revista Iberoamericana de Educación. (73). 2017. Disponible en https://rieoei.org/RIE/article/view/289

MINISTERIO DE RELACIONES EXTERIORES. (2019). Comisión ODS Paraguay 2030. Disponible en http://comisionods.mre.gov.py/

OJEDA SUAREZ; Rafael., AGÜERO CONTRERAS, Fernando Carlos. Globalización, Agenda 2030 e imperativo de la educación superior: reflexiones. Revista Conrado 15 (67) $\quad$ - $2019 . \quad$ Disponible en http://scielo.sld.cu/scielo.php?script=sci_arttext\&pid=S199086442019000200125\#f1

PACTO GLOBAL. Red Paraguay. Pacto Global Paraguay. Disponible en http://pactoglobal.org.py/ 
PNUD. Paraguay. Pacto Global. Disponible en https://www.py.undp.org/content/paraguay/es/h5ome/operations/projects/de mocratic_governance/la-red-del-pacto-global.html

PRESIDENCIA DE LA REPÚBLICA DEL PARAGUAY. GABINETE SOCIAL, Paraguay (2019). Oficializarán en Paraguay la Agenda 2030 para el Desarrollo Sostenible del planeta. Disponible en https://www.gabinetesocial.gov.py/articulo/456oficializaran-en-paraguay-la-agenda-2030-para-el-desarrollo-sostenible-delplaneta.html

SDSN Australia/Pacific (2017).Cómo empezar con los ODS en las universidades edición en español una guía para las universidades, los centros de educación superior y el sector académico. Edición en español, Red Española para el Desarrollo Sostenible (REDS / SDSN-Spain). Disponible en http://reds-sdsn.es/wpcontent/uploads/2017/02/Guia-ODS-Universidades-1800301-WEB.pdf

UNESCO. Instituto de Estadística. La pobreza en el mundo podría reducirse a la mitad si todos los adultos terminaran la educación secundaria. Disponible en https://fr.unesco.org/gem-report/sites/gem-

report/files/UISgemPoveryEducation_esp_851.pdf

UNESCO. Educación Superior y Objetivos de Desarrollo Sostenible. Disponible en https://es.unesco.org/themes/educacion-superior/ods

UNIVERSIDAD NACIONAL DE ASUNCIÓN, Paraguay. La UNA fue sede del Conversatorio Juventudes ODS Paraguay 2030. Disponible en https://www.una.py/2019/la-una-fue-sede-del-conversatorio-juventudes-odsparaguay-2030/

Yale Center for Environmental Law and Policy-Universidad de Columbia. (2018). Global metrics for the environment. Disponible en https://epi.envirocenter.yale.edu/ 